\title{
Redefinition of SI Units and Its Implications
}

\author{
S. Schlamminger ${ }^{1}$, I. Yang ${ }^{2}$ and H. Kumar ${ }^{3 *}$ (D) \\ ${ }^{1}$ National Institute of Standards and Technology (NIST), Gaithersburg, USA \\ ${ }^{2}$ Korea Research Institute of Standards and Science (KRISS), Daejeon, Republic of Korea \\ ${ }^{3}$ National Institute of Technology, New Delhi, Delhi, India
}

Received: 04 December 2020 / Accepted: 04 December 2020 / Published online: 25 January 2021

(C) Metrology Society of India 2021

\begin{abstract}
The presented paper discusses the summary of the special issue consisting of 13 peer-reviewed papers regarding Redefinition of SI Units and Its Implications. The papers discuss state-of-the-art reviews and progress made as of now and ongoing progress regarding the redefinition of SI units at different National Metrology Institutes (NMIs) and academic/ research institutes across the globe. Different methodologies adopted while investigating redefinition of SI units have been discussed and summarized by the researchers, which will serve as a pivotal point in guiding the researchers in upcoming time.
\end{abstract}

Keywords: CGPM; Redefinition; SI unit; Kilogram; XRCD; Kibble balance; Metre; Optical frequency standard

\section{Introduction}

Metrology is the science of measurements. The weight and length parameters had been prominently used by the earliest human civilizations mainly for agriculture and trade. In earlier eighteenth century with the French revolution to the very recent adoption of newly redefined system of units at 26th General Conference on Weights and Measures (CGPM), simplified and uniform systems of weights and measures were developed for measurements. The International System (SI) of units is undoubtedly paramount for the scientific and industrial development and growth of any economy. There are seven SI base units: second; metre; kilogram; ampere; kelvin; mole; and candela. The SI is the hearth of metrology and acts as a tool for any scientific development in the country. The SI unit of mass is defined based on the fixed value of plank constants. There are two principal routes that have been taken to realize the SI unit of mass. In the first route, the Kibble balance is used and kilogram is defined through the fixed value of Plank constant and second route is X-ray crystal density (XRCD) method, where the fixed numerical value of Avogadro's

This is a US government work and its text is not subject to copyright protection in the USA; however, its text may be subject to foreign copyright protection 2020 . number is defined as the kilogram. The newly adopted definition of the kilogram is particularly important because it stamps out the need of any physical artefact. The SI unit of current is defined based on the fixed value of elementary charge. The realization of the SI unit of thermodynamic temperature is defined based on the fixed numerical value of Boltzmann constant. There are various methods, such as dielectric constant gas thermometry; acoustic gas thermometry; Doppler broadening technique and noise thermometry, that have been carried out in various NMIs across the globe for realizing the thermodynamic temperature. The SI unit of luminous intensity is defined based on the fixed numerical value of luminous efficacy. The candela is defined as the luminous intensity in a given direction, which is described as taking the fixed numerical value of the luminous efficacy of monochromatic radiation of frequency $540 \times 10^{12} \mathrm{~Hz}, K_{\mathrm{cd}}$, to be 683 , when expressed in the unit

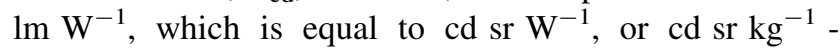
$\mathrm{m}^{-2} \mathrm{~s}^{3}$, where the kilogram, metre and second are defined in terms of $h, c$ and $\Delta v_{\mathrm{CS}}$. The SI unit of amount of substance is defined based on the fixed numerical value of Avogadro constant. The 26th meeting of CGPM acts as the paradigm shift in metrology. The latest redefinition has huge significance in the area of measurements where dependences in artefact-based standard are now changed with fundamental constants of nature.

*Corresponding author, E-mail: harishkumar@nitdelhi.ac.in 


\section{Summary}

The first/feature article is authored by Rab et al. of CSIRNPL, India. This article addressed the different stages of advancement of the measurement system and SI units in India. This study focused on the significant role of metrology and new SI system in different domains of manufacturing and service sectors so that it can establish a strong traceability chain for augmenting the industrial growth and strengthening the national quality infrastructure of India. From time to time, there have been different changes undergone in the measurement systems. It also briefly described the current status of SI unit system based on quantum metrology and related to fundamental constant [1].

The second article is authored by Kuramoto et al. from National Institute of Advanced Industrial Science and Technology, Tsukuba, Ibaraki, Japan. This study focused on the realization of new definition of Kilogram established on 20 May 2019. This new definition is explored at NMIJ by the XRCD method using ${ }^{28} \mathrm{Si}$-enriched spheres and experiment reveals that relative standard uncertainty is found to be $2.4 \times 10^{-8}$, for the two spheres of approximate $1 \mathrm{~kg}$ [2].

The third article is authored by Pramann et al. of Physikalisch-Technische Bundesanstalt (PTB), Bundesallee Braunschweig, Germany. In this article, XRCD method is used for the dissemination of the SI base unit kilogram yielding smallest possible uncertainties associated with the mass $m$ within a few parts in $10^{-8}$. This work compares different available and newly developed analytical methods and their results for the determination of the molar mass $M$ of silicon highly enriched in ${ }^{28} \mathrm{Si}(\mathrm{Me})$ and of silicon $(M \mathrm{x})$ with an almost natural isotopic distribution [3].

The fourth article is authored by Massa et al. from Istituto Nazionale di Ricerca Metrologica (INRiM), Italy. This study addressed the measurements of the spacing of the silicon planes and describes the most critical features of combined X-ray and optical interferometry. Advancement of combined X-ray and optical interferometry as applied to the metrology of nanostructures and the manipulation of matter on the atomic scale are analysed. The results depict that the numerical and experimental determinations of the surface stress are challenging. Lattice strains and lattice parameter of measurements are underway to work out the problem of phase topography [4].

The fifth article is by authored Acharya et al. of CSIRNPL, India. This article addressed the detection probe laser beams, geometry of the detection chamber, data acquisition/ processing techniques of the cold atoms in CsF1 primary standard at CSIR-NPL. For data acquisition, two distinctly separated detection channels are used to improve
$\mathrm{S} / \mathrm{N}$ ratio, resulting in efficient design of detection hardware/software [5].

The sixth article is authored by Sharma et al. of CSIRNPL, India. This article reported a systematic review of the development of optical atomic clock. These clocks operate at optical frequencies and provide better accuracy/higher stability. This article also discussed the different atomic clocks, optical frequency standards and the recent developments of atomic clocks CSIR-NPL. With the most accuracy, these optical clocks may lead to a possible redefinition of time and frequency SI unit in near future [6].

The seventh article is authored by Nandita et al. of Amrita School of Engineering, India, which discusses the atomic clock Ensemble Algorithm based on the ANN model for a time-scale. This work formulated 3, 4 and 5 clock ensemble models and it used four deviation functions to compute the deviation for the combination of each pair of clocks. The result depicts that ANN model shows the better stability when compared with other model like Kalman filter and multi-scale ensemble formation (METS) algorithm [7].

The eighth article is authored by Monika et al. of CSIRNPL, India. This article discussed the Rydberg atom-based technique for E-field strength evaluation at microwave frequency. In this study, different situations like impact of power variation of microwave, the detuning of microwave frequency and the distance variation of source antenna are considered to sense the RF electrometry and measure the E-field strength. The Rydberg atoms provide high sensitivity to the microwave radiation, detecting weak field of $12.5 \mu \mathrm{W}$ at $15.09 \mathrm{GHz}$. From the results obtained, with large frequency range, high sensitivity and self-calibration of Rydberg atoms technique are the best way for new generation E-field probes, which are traceable to SI units [8].

The ninth article is authored by Saha et al. of CSIRNPL, India. This article reported the evolution of unit of Candela and also describes the requirement of Quantum Candela. Candela is one of the seven base units, which is the only base unit to be related to the human physiology and gives a physical base to the phenomenon of vision. The advancement of semiconductor technology led to the growth of detectors opened the way for detector-based photometry [9].

The tenth article is authored by Sharma et al. of CSIRNPL, India. This article describes the redefinition of SI unit at CSIR-NPL-India from the period of Vedic civilization to the present system of SI unit of length 'Metre'. This article also describes the evolution of dimensional metrology since 1953, parallel to the realization of SI unit, including the contributions of CSIR-NPL in that field of dimensional metrology and advanced research like Shear interferometer, Jamin interferometer for surface roughness. This study 
also discussed the advanced services to be done by length, dimension and nano-metrology at CSIR-NPL [10].

The eleventh article is authored by Ehtesham et al. from of CSIR-NPL, India. In this study, author reviewed the key developments in the evolution of mass standard till now. This article discussed the working principle of Kibble balance to realize of kilogram to its new definition. Presently, NPL UK demonstrated single-mode two-measurement phase Kibble balance. The new concept opens up interesting advances in mass metrology, including various realization methods, realizing the unit (other than $1 \mathrm{~kg}$ ) and traceability sources [11].

The twelfth article is authored by Thakur et al. of CSIRNPL, India. This article focused quantum Pascal pressure standards using an optical interferometer manometer. Recently, NIST USA developed a quantum pressure standard in the $1 \mathrm{~Pa}$ to $180 \mathrm{kPa}$ pressure range. The study depicts the realization of quantum Pascal with the help of changes in the resonance frequency of EM waves during the light-matter interaction [12].

The thirteenth article is authored by Garg et al. of CSIRNPL, India. This article focused on the two particular features: measurement methodology and measurement uncertainty in realization and dissemination of unit watt in airborne sound. The dissemination of quantity sound power is usually done with a transfer standard that is commercially available aerodynamic. The traceability chain of sound power level originates with primary sound power source for realization of the unit. The development of a primary sound power standard is the utmost requirement so as to strengthen the traceability chain [13].

\section{References}

[1] S. Rab, S. Yadav, N. Garg, S. Rajput and D.K. Aswal, Evolution of Measurement System and SI Units in India. MAPAN (2020). https://doi.org/10.1007/s12647-020-00400

[2] N. Kuramoto, S. Mizushima, L. Zhang, K. Fujita, Y. Ota, S. Okubo and H. Inaba, Realization of the New Kilogram Using ${ }^{28} \mathrm{Si}$-Enriched Spheres and Dissemination of Mass Standards at NMIJ. MAPAN (2020). https://doi.org/10.1007/s12647-02000393-2
[3] A. Pramann, J. Vogl and O. Rienitz, The Uncertainty Paradox: Molar Mass of Enriched Versus Natural Silicon Used in the XRCD Method. MAPAN (2020). https://doi.org/10.1007/ s12647-020-00408-y

[4] E. Massa, C.P. Sasso and G. Mana, The Measurement of the Silicon Lattice Parameter and the Count of Atoms to Realise the Kilogram. MAPAN (2020). https://doi.org/10.1007/ s12647-020-00409-x

[5] A. Acharya, P. Arora, S. Yadav and A.S. Gupta, Detection, Acquisition and Processing of Fluorescence from Cold Atoms in Cesium Fountain Primary Frequency Standard at NPL, India. MAPAN (2020). https://doi.org/10.1007/s12647-020-00405-1

[6] L. Sharma, H.K. Rathore, S. Utreja, N. Yadav, A. Roy, S. De and S. Panja, Optical Atomic Clocks for Redefining SI Units of Time and Frequency. MAPAN (2020). https://doi.org/10.1007/ s12647-020-00397-y

[7] R. Sruthikeerthi Nandita, S. Maharana, B. Rajathilagam, T. Subramanya Ganesh and S. Krishnamoorthy. An Artificial Neural Network Model for Timescale Atomic Clock Ensemble Algorithm. MAPAN (2020). https://doi.org/10.1007/ s12647-020-00414-0

[8] Monika, H.S. Rawat and S.K. Dubey, RF E-Field Sensing Using Rydberg Atom-Based Microwave Electrometry. MAPAN (2020). https://doi.org/10.1007/s12647-020-00404-2

[9] S. Saha, V. K. Jaiswal, P. Sharma and D. K. Aswal, Evolution of SI Base Unit Candela: Quantifying the Light Perception of Human Eye. MAPAN (2020). https://doi.org/10.1007/s12647020-00413-1

[10] R. Sharma, G. Moona and M. Jewariya, Progress Towards the Establishment of Various Redefinition of SI Unit "Metre" at CSIR-National Physical Laboratory-India and Its Realization. MAPAN (2020). https://doi.org/10.1007/s12647-020-00418-w

[11] B. Ehtesham, T. John, S. Yadav, H. Krishna Singh, G. Mandal and N. Singh, Journey of Kilogram from Physical Constant to Universal Physical Constant $(h)$ Via Artefact: A Brief Review. MAPAN (2020). https://doi.org/10.1007/s12647-020-00392-3

[12] V.N. Thakur, S. Yadav and A. Kumar, Realization of Quantum Pascal Using Natural Fundamental Physical Constants. MAPAN (2020). https://doi.org/10.1007/s12647-020-00411-3

[13] N. Garg, B. S. Chauhan and M. Singh, Realization and Dissemination of Unit Watt in Airborne Sound: Measurement Methodology, Sound Emission Regulations and Implications. MAPAN (2020). https://doi.org/10.1007/s12647-020-00417-x 


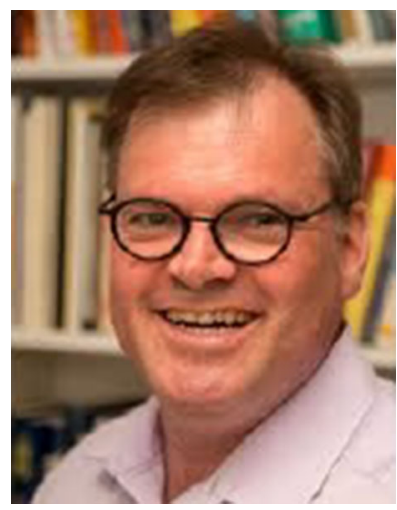

Dr. Stephan Schlamminger (M'12) received the Diploma degree in physics from the University of Regensburg, Regensburg, Germany, in 1998, and the Ph.D. degree in experimental physics from the University of Zurich, Zurich, Switzerland, in 2002. His thesis was on the determination of the Universal Constant of Gravitation. From 2002 to 2010, he was with the University of Washington, Seattle, WA, USA, where he was involved in the experimental test of the equivalence principle. In 2010, he was with the National Institute of Standards and Technology (NIST), Gaithersburg, MD, USA, where he was involved in Kibble balance. In 2016, he was a Group Leader of the Fundamental Electrical Measurement Group, NIST. From 2017 to 2018, he was with the Regensburg University of Applied Science, Regensburg, Germany, where he taught physics. Since 2018, he has been a Physicist with NIST.

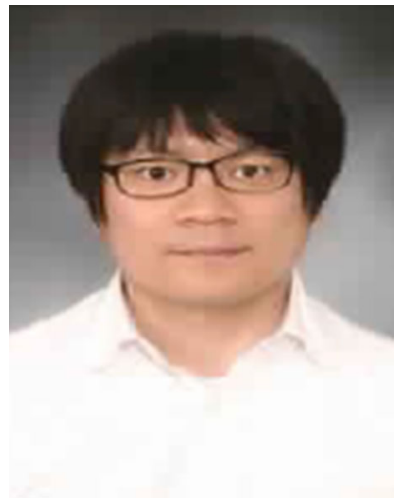

Dr. Inseok Yang received a B.S. in Physics at Korea Advanced Institute of Science and Technology (KAIST) in 1999. He joined the Korea Research Institute of Standards and Science (KRISS) in 2005, after receiving a Physics Ph.D. at the University of Chicago, USA. Since then, he has been working on thermometry, especially on the International Temperature Scale and thermodynamic temperature measurement. He was awarded the APMP (Asian Pacific Metrology Programme) Young Metrologist Award in 2013. He spent one year at the National Physical Laboratory (NPL) in UK as a guest scientist in 2017. He has authored over 70 papers in several peer-reviewed journals since 2003. He is the current Chair of the Technical Committee on Thermometry in APMP and a member of Editorial Board of Metrologia since 2017.

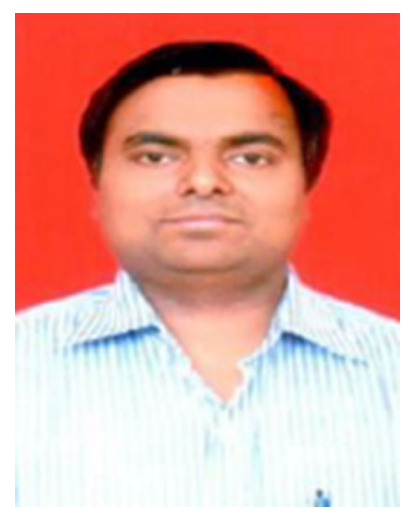

Dr. Harish Kumar received B.Tech. degree in Mechanical and Automation Engineering from the Guru Gobind Singh Indrapsatha University, New Delhi, India, in 2003 and the $\mathrm{Ph} . \mathrm{D}$. degree in Mechanical Engineering from Guru Gobind Singh Indrapsatha University, New Delhi, India, in 2015. His thesis was on design and development of force transducers. He worked at National Physical Laboratory, New Delhi, India during 2007 to 2017 as Scientist and worked in the force and mass metrology. He has worked in the design and development of Kibble balance. Since 2017, he has been working at National Institute of Technology Delhi, India and currently working in additive manufacturing in addition to metrology.

Publisher's Note Springer Nature remains neutral with regard to jurisdictional claims in published maps and institutional affiliations. 